\title{
Variables socioeconómicas de los miembros del APEC: 2000
}

$\mathrm{L}$ a estructura socioeconómica de las economías que conforman el foro de Cooperación Económica de Asia Pacífico (APEC) exponen, por su diversidad, un carácter singular. Éstas convergen ante una serie de acuerdos, principios y normas que las ha llevado, a lo largo de catorce años, a avanzar hacia la liberalización del comercio y la inversión, lo que les ha permitido facilitar la cooperación técnica y económica. Dentro del grupo se encuentran no sólo países con legados culturales diferentes, ${ }^{1}$ sino también economías con desiguales grados de desarrollo económico que, según la clasificación del Banco Mundial, ${ }^{2}$ oscilan entre economías de desarrollo bajo, medio y alto.

De acuerdo con Simona Vera y Anne Thompson (1995: 16), el desarrollo está conformado por cuatro elementos:

1. Modernización: se refiere a los cambios en la estructura y en la conducta que una sociedad debe experimentar en el proceso de la adquisición de un sistema industrial de producción y distribución.

2. Tecnología: el desarrollo requiere de conocimientos y de un mayor uso de maquinaria compleja y sofisticada.

3. Organización: implica el desarrollo de la creciente especialización en las instituciones políticas, sociales y económicas, así como en los múltiples roles que se imponen sobre el ciudadano.

4. Conducta: el desarrollo involucra un amplio rango de nuevas creencias y actitudes que el individuo debe de adquirir para ajustarse a las demandas colocadas sobre ellos por la movi-

\footnotetext{
* Estudiante de la Maestría en Ciencias Sociales, Universidad de Guadalajara.

ORCID http://orcid.org/0000-0002-4553-8141
}

\author{
lidad geográfica y social, la amplitud \\ de la legalidad política, del crecimien- \\ to económico y la tecnología.
}

El objetivo de esta contribución es dar al lector una visión general sobre los principales factores económicos, sociales, demográficos y territoriales de cada uno de estos grupos de economías.

\section{Economías con desarrollo alto}

Diez de las 21 economías que conforman el APEC se distinguen por tener un nivel de desarrollo alto, los habitantes de Estados Unidos, Canadá, Japón, Australia, Hong Kong, Singapur, Brunei, Nueva Zelanda, Corea del Sur y Taiwan obtienen un ingreso per cápita que oscila entre 14188 a 34100 dólares internacionales. Lo que significa que en el año 2000 los 543.5 millones de personas que viven dentro de este grupo obtuvieron, en promedio, un ingreso per cápita de 22774 dólares internacionales. Cantidad que permite a sus habitantes aumentar, en general, su calidad de vida al tener acceso al consumo de artículos de lujo, a la educación, información, salud, recreación, cuidado ambiental, entre otros. ${ }^{3}$

De acuerdo con Samuel Huntington (1985: 170-171) las economías desarrolladas se caracterizan por tener un gobierno político racional; por emplear políticas modernas que incluyan la diferenciación de las nuevas funciones políticas, y por el desarrollo de estructuras especializadas para ejecutar tales funciones en áreas de competencia legal, militar, administrativa y científica; además del incremento de la participación ciudadana en asuntos políticos y sociales.

S. Eisenstadt (1992: 338) afirma que las sociedades desarrolladas se caracterizan por 
contar con modelos de acción que les permiten acrecentar la calidad de vida de sus habitantes en salud, educación, empleo, actividades culturales y recreativas, y agrega que el sistema económico de estos países se centra en el sector secundario y de servicios.

Es importante señalar que, con excepción de Brunei (país cuya actividad económica se centra en el petróleo, casi con el $50 \%$ del PIB), la estructura económica del resto de las economías coincide con la afirmación anterior, ya que la contribución al producto interno bruto (PIB) del sector industrial y de servicios representa alrededor del $96 \%$, mientras que el sector agrícola aporta el resto, incluso en economías como Singapur y Hong Kong la producción agrícola es prácticamente una actividad nula. La situación difiere en Nueva Zelanda, ya que este país se distingue por el eficiente manejo de su sistema agrícola, lo que le permitió contribuir con un $7 \%$ al PIB en el año 2000.

A pesar del desarrollo económico alcanzado, la distribución del ingreso no es equitativa en todas las economías de este grupo. La brecha de consumo del $20 \%$ de las familias más ricas de la población respecto al $20 \%$ de las familias más pobres es muy amplia en algunos. Dentro del grupo, Hong Kong, Nueva Zelanda, Estados Unidos y Australia tienen una distribución del ingreso menos equitativa. En Hong Kong $60 \%$ del ingreso lo ostenta el $20 \%$ más rico, mientras el $20 \%$ más pobre abarca sólo el $4.4 \%$. En las otras tres economías la renta del $20 \%$ más rico absorbe entre 41.3 y $46.9 \%$, y el consumo de los más pobres se encuentra entre el 2.7 y $5.9 \%$.

Por otra parte, Canadá y Corea poseen una distribución del ingreso más equitativa y el $20 \%$ de las familias más ricas absorbe el 39.3 del ingreso nacional y el $20 \%$ de los más pobres detenta el $7.5 \%$ de la riqueza. Dentro del grupo destaca Japón, por presentar indicadores de distribución más favorables y equitativos para el conjunto de su población.

Así, el ingreso de las familias de más altos ingresos es tres veces mayor que el de las familias de más bajos ingresos. Las familias niponas gozan de mejores estándares de vida no sólo en este grupo sino en todas las economías del APEC. Esto último se confirma con el coeficiente de Gini, ${ }^{4}$ indicador que mide el nivel de desigualdad económica de un país, en donde Japón se encuentra mejor posicionado que todos los miembros del APEC, con un 24.9 en contraste con Hong Kong, que tiene 52.2 puntos en la escala de Gini.

El capital físico siempre se ha encontrado en el centro de las explicaciones del progreso económico. Para invertir, un país debe ahorrar o tener acceso al ahorro extranjero por medio de préstamos o ayudas (Dornbusch y Fischer, y Schmalensee, 1990: 558-560). Por lo que el ahorro interno juega un papel muy importante en el desarrollo. Dentro del grupo, por sus altas tasas de ahorro destaca Singapur con $51.5 \%$ respecto al PIB de 2000 , le siguen Hong Kong y Japón con 32.5 y $29.4 \%$, respectivamente. Del mismo modo, estas tres economías presentan los mayores porcentajes de inversión, alcanzando el 31, 28 y $26 \%$ del PIB.

La actividad comercial alcanzada por las economías del APEC la coloca como la región más activa, ya que en la actualidad tiene una participación de $47 \%$ en el comercio mundial total. Todas las economías se han propuesto desarrollar estrategias para alcanzar las metas de Bogor, ${ }^{5}$ en las cuales se plantea que para 2010 el libre comercio e inversión en Asia Pacífico será posible para las economías desarrolladas, mientras que para los países en vías de 
Cuadro 1

APEC: economías con desarrollo alto, 2000

\begin{tabular}{|c|c|c|c|c|c|c|c|c|c|c|}
\hline Indicadores & $\begin{array}{l}\text { Estados } \\
\text { Unidos }\end{array}$ & Canadá & Japón & Australia & Hong Kong & Singapur & Brunei & \begin{tabular}{|c|} 
Nueva \\
Zelanda \\
\end{tabular} & Corea & Taiwan \\
\hline 1. Territorio, miles $\mathrm{km}^{2}$ & $9,159.1$ & $9,221.0$ & 376.5 & $7,682.3$ & 1.0 & 0.6 & 442.0 & 268.0 & 120.4 & 36.0 \\
\hline Forestal, porcentaje 2000 & 24.7 & 26.5 & 66.1 & 20.6 & .. & 3.3 & $83.87^{\star}$ & 29.7 & 63.3 & 58.6 \\
\hline Promedio anual de deforestación, 1990-2000 (\%) & -0.2 & $0.0^{\star \star}$ & 0.0 & -0.2 & .. & 0.0 & 0.2 & -0.5 & 0.1 & N.D. \\
\hline 2. Población, miles & $282,000.0$ & $31,000.0$ & $126,870.0$ & $19,000.0$ & $7,000.0$ & $4,000.0$ & 331.0 & $4,000.0$ & $47,000.0$ & $22,276.0$ \\
\hline Habitantes por $\mathrm{km}^{2}$, personas & 31.0 & 3.0 & 337.0 & 2.0 & & $6,587.0$ & 58.3 & 14.0 & 479.0 & 616.0 \\
\hline Esperanza de vida al nacer, años & 77.0 & 79.0 & 81.0 & 79.0 & 80.0 & 78.0 & 74.8 & 78.0 & 73.0 & 75.3 \\
\hline Tasa de mortalidad infantil, por 1000 nacidos vivos & 7.0 & 5.0 & 4.0 & 5.0 & 3.0 & 3.0 & 13.9 & 6.0 & 8.0 & 5.9 \\
\hline Mujer en la fuerza de trabajo, (\%) del total & 46.0 & 45.8 & 41.1 & 43.7 & 37.1 & 39.1 & 55.1 & 45.0 & 41.4 & 46.0 \\
\hline Población mayor de 65 años, (\%) del total & 12.3 & 12.6 & 17.2 & 12.3 & 10.6 & 7.2 & 3.d & 11.7 & 7.1 & 8.6 \\
\hline Promedio de años de escolaridad & 12.0 & 11.6 & 9.5 & 10.9 & 9.4 & 7.0 & N.D & 11.7 & 10.8 & N.D. \\
\hline \multicolumn{11}{|l|}{ 3. Economía } \\
\hline Ingreso Nacional Bruto (INB), mill. dls. & $9,601,500.0$ & $649,800.0$ & $4,519,067.0$ & $388,300.0$ & $176,200.0$ & $99,400.0$ & $4,600 . d$ & $49,800.0$ & $421,100.0$ & $309,426.0$ \\
\hline INB per cápita, dólares & $34,100.0$ & $21,130.0$ & $35,620.0$ & $20,240.0$ & $25,920.0$ & $24,740.0$ & $13,897.3$ & $12,990.0$ & $8,910.0$ & $14,188.0$ \\
\hline INB per cápita, dólares internacionales & $34,100.0$ & $27,170.0$ & $27,080.0$ & $24,970.0$ & $25,590.0$ & $24,910.0$ & $13,897.3^{\mathrm{a}}$ & $18,530.0$ & $17,300.0$ & $14,188.0^{\mathrm{a}}$ \\
\hline Crecimiento promedio anual (1999-2000), (\%) & 4.2 & 4.5 & 2.4 & 1.9 & 10.5 & 9.9 & 7.6 & 2.5 & 8.8 & 7.5 \\
\hline \multicolumn{11}{|l|}{ Estructura del PIB (\%) (1990 y 2000) } \\
\hline Agricultura & N.D. & 3 & 2 y 1 & 3 у 3 & 0 y 0 & 0 y 0 & 5 & 7 & 9 y 5 & 4 y 2 \\
\hline Industria & N.D. & 33 & 39 у 32 & 28 y 26 & 25 y 14 & 34 у 34 & 45 & 28 & 43 y 43 & 41 y 32 \\
\hline Servicios & N.D. & 64 & 58 y 66 & 68 y 71 & 74 y 85 & 65 y 66 & 50 & 65 & 48 y53 & 55 y 66 \\
\hline Población activa, cifras en miles & $144,700.0$ & $16,500.0$ & $67,410.0$ & $9,800.0$ & $3,600.0$ & $2,000.0$ & N.D & $1,900.0$ & $24,200.0$ & $9,784.0$ \\
\hline \multicolumn{11}{|l|}{ En la agricultura, porcentaje (\%) } \\
\hline Hombres & 4.0 & 5.0 & 5.0 & 6.0 & $0^{a}$ & $0^{\mathrm{a}}$ & N.D & N.D. & 10.0 & N.D. \\
\hline Mujeres & 1.0 & 2.0 & 6.0 & 4.0 & $0^{a}$ & $0^{\mathrm{a}}$ & N.D & N.D. & 13.0 & N.D. \\
\hline \multicolumn{11}{|l|}{ En la industria, porcentaje (\%) } \\
\hline Hombres & 32.0 & 32.0 & 38.0 & 30.0 & 28.0 & 33.0 & N.D & 32.0 & 34.0 & N.D. \\
\hline Mujeres & 12.0 & 11.0 & 22.0 & 10.0 & 12.0 & 23.0 & N.D & 12.0 & 19.0 & N.D. \\
\hline \multicolumn{11}{|l|}{ En los servicios, porcentaje (\%) } \\
\hline Hombres & 64.0 & 63.0 & 57.0 & 64.0 & 71.0 & 67.0 & N.D & 56.0 & 56.0 & N.D. \\
\hline Mujeres & 86.0 & 87.0 & 73.0 & 86.0 & 88.0 & 77.0 & N.D & 81.0 & 68.0 & N.D. \\
\hline Desempleo & 4.0 & 6.8 & 4.7 & 6.3 & N.D: & N.D. & N.D & 6.0 & 4.1 & N.D. \\
\hline Costo por trabajador en manufactura (dólares por año) (1995-1999) & $28,907.0$ & $28,424.0$ & $31,687.0$ & $26,087.0$ & $10,353.0$ & $21,317.0$ & N.D & $18,419.0$ & $10,743.0$ & N.D. \\
\hline Inversión en la economía, porcentaje del PIB (\%) & 21.0 & 20.0 & 26.0 & 24.0 & 28.0 & 31.0 & 14.3 & 21.0 & 29.0 & 22.6 \\
\hline Tasa de ahorro bruta, porcentaje del PIB & 18.0 & 25.3 & 29.4 & 18.9 & 32.5 & 51.5 & N.D & 18.6 & 31.3 & 25.4 \\
\hline Inflación promedio anual, 1990-2000 (\%) & 2.1 & 1.4 & 0.1 & 1.5 & 4.1 & 1.3 & 1.1 & 1.5 & 5.0 & N.D. \\
\hline \multicolumn{11}{|l|}{ 4. Sector externo } \\
\hline Exportaciones, millones de dólares & $781,125.0$ & $276,635.0$ & $479,283.0$ & $63,869.0$ & $202,440.0$ & $137,875.0$ & $3,000.0$ & $13,267.0$ & $172,268.0$ & $148,321.0$ \\
\hline Exportaciones manufacturales como \% del total & 83.0 & 64.0 & 98.8 & 29.0 & 95.0 & 86.0 & N.D & 28.0 & 91.0 & 98.6 \\
\hline Exportaciones en el PIB (\%) & 11.0 & 44.0 & 10.0 & 20.0 & 150.0 & 180.0 & N.D & 32.0 & 45.0 & 44.0 \\
\hline Crecimiento promedio anual (\%) (1990-1999) & 6.7 & 9.1 & 1.9 & 7.5 & 8.8 & 13.5 & N.D & 4.6 & 15.3 & N.D. \\
\hline Importaciones, millones de dólares & $1,257,636.0$ & $244,786.0$ & $379,718.0$ & $71,531.0$ & $214,200.0$ & $134,545.0$ & $1,400.0$ & $13,906.0$ & $160,481.0$ & $140,011.0$ \\
\hline Importación de alimentos, \% del total de importaciones & 4.0 & 5.0 & 12.1 & 5.0 & 4.0 & 3.0 & 21.0 & 8.0 & 5.0 & N.D. \\
\hline Importaciones en el PIB (\%) & 13.0 & 41.0 & 8.0 & 22.0 & 145.0 & 161.0 & N.D & 33.0 & 42.0 & 52.1 \\
\hline Crecimiento promedio anual (\%) (1990-1999) & 8.9 & 9.0 & 5.2 & 9.3 & 9.5 & 9.9 & N.D & 6.2 & 9.5 & N.D. \\
\hline Deuda externa (millones) & N.D. & N.D. & N.D & N.D. & N.D & N.D. & N.D & N.D & $134,417.0$ & N.D. \\
\hline Turistas visitantes al año (miles de personas) & $50,891.0$ & $20,423.0$ & $4,757.0$ & $4,946.0$ & $13,059.0$ & $6,258.0$ & $37^{c}$ & $1,787.0$ & $5,322.0$ & $2,624.0$ \\
\hline Turistas al exterior (miles de personas) & $58,386.0$ & $18,368.0$ & $16,358.0$ & $3,210.0$ & $4,175.0$ & $3,971.0$ & N.D & $1,185.0$ & $5,508.0$ & $7,328.0$ \\
\hline Ingresos por turismo (millones) & $85,153.0$ & $10,768.0$ & $3,374.0$ & $8,442.0$ & $7,886.0$ & $6,370.0$ & $964^{\mathrm{c}}$ & $2,068.0$ & $6,609.0$ & N.D. \\
\hline \multicolumn{11}{|l|}{ 5. Otros indicadores del nivel de vida } \\
\hline Consumo de electricidad per cápita kwh (1999) & $11,994.0$ & $15,260.0$ & $7,443.0$ & $8,884.0$ & $5,178.0$ & $6,641.0$ & $7,676.0$ & $8,426.0$ & $5,160.0$ & $1,570.8$ \\
\hline Líneas telefónicas y teléfonos móviles (2000), por 1000 habitantes & $1,098.0$ & 962.0 & $1,112.0$ & 972.0 & $1,392.0$ & $1,168.0$ & N.D & $1,063.0$ & $1,031.0$ & $1,374.0$ \\
\hline Computadoras personales (2000), por 1000 habitantes & 585.2 & 390.2 & 315.2 & 464.6 & 350.6 & 483.1 & 70.1 & 360.2 & 237.9 & N.D. \\
\hline Usuarios de internet (miles) & $95,354.0$ & $12,700.0$ & $47,080.0$ & $6,600.0$ & 350.6 & $1,200.0$ & 3.9 & 830.0 & $19,040.0$ & $6,270.0$ \\
\hline Periódicos por cada mil habitantes & 213.0 & 159.0 & 578.0 & 293.0 & 792.0 & 298.0 & N.D & 207.0 & 393.0 & N.D. \\
\hline Gasto público en salud (\% del PIB) & 5.7 & 6.6 & 5.7 & 6.0 & 2.1 & 1.1 & N.D & 6.3 & 2.4 & N.D. \\
\hline Coeficiente de Gini & 40.8 & 31.5 & 24.9 & 35.2 & 52.2 & N.D & N.D & N.D. & 31.6 & 33.0 \\
\hline Ingreso o consumo del $20 \%$ más rico de la población (\%) & 46.4 & 39.2 & 35.7 & 41.3 & 57.1 & N.D. & N.D & $46.9^{\mathrm{b}}$ & 39.3 & N.D. \\
\hline Ingreso o consumo del $20 \%$ más pobre de la población (\% & 5.2 & 7.5 & 10.6 & 5.9 & 4.4 & N.D. & N.D & $2.7^{\mathrm{b}}$ & 7.5 & N.D. \\
\hline
\end{tabular}

Fuente: Banco Mundial, World Bank Atlas 2002, World Development Indicators 2002, APEC Economic Outlook 2001 y 2002. www.brunei.gov.bn/about_brunei/economy.htm., www.stat.gov.tw y www.cia.gov/cia/publications/facbook/geos/bx.html.

Notas:

En la estructura del PIB, los datos de Estados Unidos y Nueva zelanda son de 1990.

*Los datos de Brunei sobre los recursos forestales son de la FAO.

** Australia, Brunei y Singapur en la tasa anual de deforestación son datos de la FAO.

***Dato de 1999, www.brunei.gov.bn/about brunei/land.htm.

Datos de Taiwan, http://www.geo.gov.tw/taiwan-website/5-pg/yearbook/.

a menos de $0.5 ;^{\text {b }}$ datos de $1999,{ }^{c}$ datos de 1999. 
desarrollo la meta es a 2020. En el año 2000, el comercio total (importaciones más exportaciones) de las 21 economías del APEC alcanzó los 6 436320 millones de dólares, de esta cantidad casi el $52 \%$ corresponde a las importaciones. De todos los miembros del APEC, las economías con saldos deficitarios en orden ascendente son Estados Unidos, México, Hong Kong, Australia, Perú, Vietnam y Nueva Zelanda.

Dentro del grupo de las economías con desarrollo alto se encuentran algunos de los principales comercializadores del mundo: Estados Unidos, Japón, Canadá, Hong Kong, Corea del Sur, Taiwan y Singapur figuran, desde la segunda mitad del siglo XX, como el grupo más dinámico en el comercio internacional. Así, la participación de este grupo en el comercio total del APEC representó el $67 \%$ en el año 2000. Para Japón, Taiwan, Hong Kong y Corea, la mayor proporción de sus exportaciones (más del 91\%) corresponde a productos manufacturados, mientras que para Nueva Zelanda, Australia y Brunei este porcentaje no llega al 30\%. Las economías que sobresalen por tener mayores ingresos vía exportaciones, comparado con lo que se produce internamente, son Singapur y Hong Kong ya que sus exportaciones en proporción al PIB equivalen a más del doble. Por otro lado, los países que se exhiben como los grandes importadores de alimentos, con respecto al total de sus importaciones, son Brunei (21\%) y Japón (12.1\%), para este último la tasa de autosuficiencia alimentaria es muy baja (40\%), convirtiéndolo así en el mayor importador de alimentos del mundo.

En lo que se refiere a la industria turística, ésta se vislumbra como uno de los sectores con mayor dinamismo entre las economías del sistema mundial. Conscientes de lo anterior, los miembros del APEC establecieron como parte de sus prioridades a partir de 2002 promover la afluencia turística, mediante: 1 ) eliminar los impedimentos al turista por negocios e inversión; 2) incrementar la movilidad de visitantes y la demanda de bienes y servicios turísticos; 3) impulsar el manejo sustentable del turismo, y 4) reconocer al turismo como un vínculo para el desarrollo social y económico. ${ }^{6}$

Según la Organización Mundial del Turismo (OMT), el APEC incluye seis de los quince países con mayor captación de divisas por turismo: Estados Unidos, Canadá, Australia, China, México y Hong Kong. De los 474 millones de dólares que se generaron a nivel mundial en este rubro durante el año 2000, los países del APEC captaron el 39\%, mientras que el grupo de las economías con desarrollo alto obtuvo el $27.6 \%{ }^{7}$

En el aspecto social, según el Programa de las Naciones Unidas para el Desarrollo (PNUD), el desarrollo humano debe entenderse como el proceso de ampliación de las opciones de la gente, aumentando las funciones y las capacidades más elementales, como la de vivir una vida larga y saludable, estar bien informado y disfrutar de un nivel de vida digno (PNUD, 2000: 17).

En este sentido, resulta inevitable reconocer que aun dentro del grupo de los países con desarrollo económico alto falta mucho por hacer en cuanto al desarrollo social. En promedio los ciudadanos de este grupo tienen una esperanza de vida de 77.5 años, destacando el caso de Japón ${ }^{8}$ y de Hong Kong por ser los que presentan el índice más alto (81 y 80 años, respectivamente), mientras que Brunei y Corea sobresalen por tener los más bajos $(74.8$ y 73 años). Dentro de la tasa de mortalidad infantil por cada mil niños nacidos, Brunei de nuevo encabeza la lista por ser el país con la mayor tasa de este grupo (13.9), le sigue Corea con una tasa de 8. En contraste, Hong Kong y Singapur se distinguen por tener la tasa de mortalidad infantil más baja, con sólo 3 de cada mil niños nacidos.

En la actualidad los medios electrónicos, como las computadoras e Internet, se han constituido en una herramienta necesaria en el proceso de aprendizaje, la obtención de información y como generadora de conocimiento. Debido a ello, el acceso de la población a estos servicios indica, por un lado, la capacidad eco- 
nómica con la que cuentan los ciudadanos $\mathrm{y}$, por otro, la libertad de acceso a los medios (Human Development Report, 2000: 73). En Estados Unidos más de la mitad de sus ciudadanos poseen computadoras personales y $38 \%$ de la población usan Internet, hecho que lo coloca con la proporción más amplia de este grupo; le siguen Singapur y Australia. La población de Brunei es la que presenta el menor acceso a computadoras personales: sólo el 7\% de su población tiene acceso a ellas y el $1.7 \%$ es usuario de Internet.

En cuanto a los años de escolaridad, con excepción de Brunei y Taiwan, de los cuales no se tienen datos, la población de este grupo tiene en promedio 10.4 años de educación formal, sobresalen Estados Unidos (12 años), Nueva Zelanda (11.7 años) y Canadá (11.6 años). Mientras que Singapur muestra el índice más bajo, con sólo 7 años de escolaridad en promedio.

\section{Economías con desarrollo medio}

Los países que conforman el bloque de las economías con desarrollo medio en el APEC, que destaca por la presencia de los tres países latinoamericanos, son: Chile, México, Malasia, Rusia, Tailandia, Perú, Filipinas y China. Este grupo se caracteriza por concentrar la mayor población de la región por la influencia de China, pues el 74\% de los 1706966 personas que pertenecen a este bloque son chinos.

Con respecto a los indicadores económicos, el ingreso per cápita de los habitantes de este grupo oscila entre 3920 y 9100 dólares internacionales, que corresponden a los ingresos de China y Chile, respectivamente. Chile presenta la mayor desigualdad en la distribución del ingreso; China muestra una relativa mejoría en la distribución de la riqueza. La relación entre el grupo de ingreso más rico en relación con el más pobre es en el caso de Chile de 18.5 veces, mientras que en China es de 7.9 veces.

De acuerdo con la estructura del PIB, en la mayoría de las economías, excepto Chile y
Perú, la participación de la agricultura disminuye considerablemente de 1990 a 2000. El caso más dramático es el de Rusia, donde en el transcurso de diez años disminuyó 10 puntos porcentuales, que fueron absorbidos por el sector servicios. China también mostró una disminución promedio del 9\%, aunque, a diferencia de Rusia, el reacomodo se dio en el sector industrial. Así, la mayor parte de la población económicamente activa se encuentra en el sector servicios y en el sector industrial.

La inversión y el ahorro en este grupo presentan un promedio de 23 y $30 \%$ en relación con el PIB, destacando con los mayores porcentajes de inversión Malasia (26\%) y con la proporción más baja Rusia (17\%). Por el lado de la tasa de ahorro sobresale de nuevo Malasia; al tener la tasa de ahorro más alta del grupo (42.2\%) y los ciudadanos de Perú con la tasa de ahorro más baja (17.8\%).

En el sector externo, este grupo de países presenta un superávit en su balanza comercial, con un saldo positivo de 95117 millones de dólares. El mayor comercializador del conjunto es China (249 297 millones de dólares en exportaciones y 225097 en importaciones); le siguen en importancia México y Malasia. En la mayoría de estas economías las exportaciones están constituidas por productos manufacturados, excepto en Chile, Perú y Rusia. Las economías con una mayor dependencia de las exportaciones son Malasia, Tailandia y Filipinas; en el primero representan casi el doble del PIв (125\%), en el segundo 67\% y en el tercero equivalen a poco más de la mitad del PIB (56\%).

En cuanto a las importaciones para el grupo en su conjunto, durante 2000 alcanzaron los 658000 millones de dólares; los mayores importadores fueron China y México, entre los dos representaron el $62 \%$ de las importaciones totales del grupo. Al igual que en las exportaciones, las economías con mayores importaciones respecto al PIB son Malasia, Tailandia y Filipinas; en el primero la cifra dobla el PIB nacional, mientras que para las 
Cuadro 2

APEC: economías con desarrollo alto medio, 2000

\begin{tabular}{|c|c|c|c|c|c|c|c|c|}
\hline & Chile & México & Malaisia & Rusia & Tailandia & Perú & Filipinas & China \\
\hline 1. Territorio, miles $\mathrm{km}^{2}$ & 748.8 & $1,908.7$ & 328.6 & $16,888.5$ & 510.9 & $1,280.0$ & 298.2 & 9,32 \\
\hline Forestal, porcentaje 2000 & 20.7 & 28.9 & 58.7 & 50.4 & 28.9 & 50.9 & 19.4 & 17. \\
\hline Promedio anual de deforestación 1990-2000 (\%) & 0.1 & 1.1 & 1.2 & 0 & 0.7 & 0.4 & 1.4 & -1 . \\
\hline 2. Población, miles & $15,000.0$ & $97,966.0$ & $23,000.0$ & $146,000.0$ & $61,000.0$ & $26,000.0$ & $76,000.0$ & $1,262,000$ \\
\hline Habitantes por $\mathrm{km}^{2}$, personas & 20 & 51.33 & 71 & 9 & 119 & 20 & 253 & \\
\hline Esperanza de vida al nacer, años & 76 & 73 & 73 & 65 & 69 & 69 & 69 & \\
\hline Tasa de mortalidad infantil, por 1000 nacidos vivos & 10 & 29 & 8 & 16 & 28 & 32 & 31 & \\
\hline Mujer en la fuerza de trabajo (\%) del total & 33.6 & 33.2 & 37.9 & 49.2 & 46.3 & 31.3 & 37.8 & 45. \\
\hline Población mayor de 65 años, (\%) del total & 7.2 & 4.7 & 4.1 & 12.5 & 5.2 & 4.8 & 3.5 & \\
\hline Promedio de años de escolaridad & 7.5 & 7.2 & 6.8 & & 6.5 & 7.6 & 8.2 & \\
\hline \multicolumn{9}{|l|}{ 3. Economía } \\
\hline Ingreso Nacional Bruto (INB), mill. dls. & $69,800.0$ & $497,025.0$ & $78,700.0$ & $241,000.0$ & $121,600.0$ & $53,400.0$ & $78,800.0$ & $1,062,900.0$ \\
\hline INB per cápita, dólares & $4,590.0$ & $5,070.0$ & $3,380.0$ & $1,660.0$ & $2,000.0$ & $2,080.0$ & $1,040.0$ & 840.0 \\
\hline INB per cápita, dólares internacionales & $9,100.0$ & $8,790.0$ & $8,330.0$ & $8,010.0$ & $6,320.0$ & $4,660.0$ & $4,220.0$ & $3,920.0$ \\
\hline Crecimiento promedio anual (1999-2000) (\%) & 5.4 & 6.9 & 8.3 & 8.3 & 4.3 & 3.1 & 4 & \\
\hline \multicolumn{9}{|l|}{ Estructura del Producto Interno Bruto (PIB) (\%) (1990 y 2000) } \\
\hline Agricultura & 9 y 11 & 8 y 4 & 15 y 11 & 17 y 7 & $12 \mathrm{y} 10$ & 7 y 8 & 22 y 16 & 27 y 16 \\
\hline Industria & 41 y 34 & 28 y 28 & 42 y 45 & 48 y 39 & 37 y 40 & 23 у 27 & 34 y 31 & 42 y 51 \\
\hline Servicios & 50 y 56 & 64 y 67 & 43 y 44 & 35 y 54 & 50 y49 & 70 y 65 & 44 y 53 & 31 у 33 \\
\hline Población activa, cifras en miles & $6,200.0$ & $39,507.0$ & $9,600.0$ & $77,700.0$ & $36,800.0$ & $9,700.0$ & $31,900.0$ & $756,800.0$ \\
\hline \multicolumn{9}{|l|}{ En la agricultura $(\%)$} \\
\hline Hombres & 19 & 27.0 & 21 & 15 & 50 & 8 & 47 & N.D \\
\hline Mujeres & 5 & 9.0 & 13 & 8 & 47 & 3 & 27 & N.D \\
\hline \multicolumn{9}{|l|}{ En la industria (\%) } \\
\hline Hombres & 31 & 27.0 & 33 & 36 & 20 & 25 & 18 & N.D \\
\hline Mujeres & 14 & 21.0 & 29 & 23 & 17 & 11 & 13 & N.D \\
\hline \multicolumn{9}{|l|}{ En los servicios (\%) } \\
\hline Hombres & 49 & 45 & 46 & 49 & 31 & 67 & 36 & N.D. \\
\hline Mujeres & 82 & 69 & 58 & 69 & 36 & 86 & 61 & N.D. \\
\hline Costo por trabajador en manufactura (dólares por año) (1995-1999) & $5,822.0$ & $7,607.0$ & $3,429.0$ & $15,528.0$ & $3,868.0$ & & $2,450.0$ & 729.0 \\
\hline Inversión en la economía, porcentaje del PIB (\%) & 23 & 23 & 26 & 17 & 23 & 20 & 18 & \\
\hline Tasa de ahorro bruta, porcentaje del PIB & 22.6 & 20.7 & 42.2 & 35.4 & 30.3 & 17.8 & 28.9 & 39.7 \\
\hline Inflación promedio anual, 1990-2000 (\%) & 7.3 & 18.9 & 3.9 & 162 & 4.2 & 26.8 & 8.4 & \\
\hline \multicolumn{9}{|l|}{ 4. Sector externo } \\
\hline Exportaciones, millones de dólares & $18,158.0$ & $166,424.0$ & $98,237.0$ & $105,200.0$ & $69,057.0$ & $7,002.0$ & $39,783.0$ & $249,297.0$ \\
\hline Exportaciones manufacturales como \% del total & 16 & & 80 & & 76 & 20 & & \\
\hline Exportaciones en el PIB (\%) & 32 & 31 & 125 & 46 & 67 & 16 & 56 & \\
\hline Crecimiento promedio anual (\%) (1990-1999) & 10.3 & 15.4 & 15.8 & & 4.1 & 9 & 17 & 10.5 \\
\hline Importaciones, millones de dólares & $18,070.0$ & $182,635.0$ & $82,210.0$ & $45,500.0$ & $61,924.0$ & $8,797.0$ & $33,808.0$ & 225,097 . \\
\hline Importación de alimentos \% del total de importaciones & & & 4 & 15 & 4 & 12 & & \\
\hline Importaciones en el PIB (\%) & 31 & 33 & 104 & 25 & 59 & 18 & 50 & 23 \\
\hline anual (\%) (1990-1999) & 11.4 & 12 & 11.2 & & -2.3 & 12.3 & 13.7 & \\
\hline Deuda externa (millones) & 36978 & 150,288 & 41797 & 160300 & 79675 & 28560 & 50063 & $14980 c$ \\
\hline Turistas visitantes al año (miles de personas) & $1,742.0$ & $20,643.0$ & $10,222.0$ & $21,169.0$ & $9,509.0$ & $1,027.0$ & $2,171.0$ & $31,229.0$ \\
\hline Turistas al exterior (miles de personas) & $1,567.0$ & $11,081.0$ & $26,067.0$ & $18,371.0$ & $1,909.0$ & 781.0 & $1,755.0$ & $10,473.0$ \\
\hline Ingresos por turismo (millones de dólares) & 827.0 & $8,295.0$ & $4,563.0$ & $7,510.0$ & $7,119.0$ & $1,001.0$ & $2,534.0$ & $16,231.0$ \\
\hline \multicolumn{9}{|l|}{ 5. Otros indicadores del nivel de vida } \\
\hline Consumo de electricidad per cápita kwh (1999) & $2,309.0$ & $1,570.0$ & $2,474.0$ & $4,050.0$ & $1,352.0$ & 654.0 & 454.0 & 759.0 \\
\hline Líneas telefónicas y teléfonos móviles (2000), por 1000 habitantes & 443.0 & 267.0 & 412.0 & 240.0 & 142.0 & 112.0 & 124.0 & 178.0 \\
\hline Computadoras personales (2000), por 1000 habitantes & 82.3 & 50.6 & & 42.9 & 24.3 & 40.9 & 19.3 & 15.9 \\
\hline Usuarios de internet (miles) & $2,537.0$ & $2,712.0$ & $3,700.0$ & $3,100.0$ & $2,300.0$ & $2,500.0$ & $2,000.0$ & $22,500.0$ \\
\hline Periódicos por cada $n$ & 98 & 98 & 158 & 105 & 64 & 0 & 82 & \\
\hline Gasto público en salud (\% del PIB) & 2.7 & 2.6 & 1.4 & 4.6 & 1.9 & 2.4 & 1.6 & \\
\hline Coeficiente de Gini & 56.7 & 53 & 49.2 & 48.7 & 41.4 & 46.2 & 46.2 & 40.3 \\
\hline Ingreso o consumo del $20 \%$ más rico de la población (\%) & 61 & 57 & 54.3 & 53.7 & 48.4 & 51.2 & 52.3 & 46.6 \\
\hline Ingreso o consumo del $20 \%$ más pobre de la población (\%) & 3.3 & 4 & 4.4 & 8.6 & 6.4 & 4.4 & 5.4 & \\
\hline
\end{tabular}

Fuente: Banco Mundial, World Bank Atlas 2002, World Development Indicators 2002, APEC Economic Outlook 2001 y 2002. www.brunei.gov.bn/about_brunei/economy.htm; www.stat.gov.tw; www.cia.gov/cia/publications/facbook/geos/bx.html.

Nota: N.D. = no disponible 
otras dos la proporción oscila entre 60 y $50 \%$. En México éstas representan sólo el 33\%. Los países que mayores cantidades de alimentos demandan del exterior, con relación al PIB, son Rusia (15\%) y Perú (12\%); en el resto el porcentaje no sobrepasa el $8 \%$.

Para terminar con el comportamiento del comercio exterior en este grupo, durante la década de los noventa los intercambios comerciales crecieron, por el lado de las exportaciones, un promedio del $12 \%$ y por el de las importaciones fue de $9.7 \%$. Cabe destacar que el crecimiento de las importaciones de Tailandia, durante los noventa, fue negativo.

Una de las características de este grupo es la elevada deuda externa que tienen todas las economías, la cual alcanza una suma total de 658041 millones de dólares para el grupo. Los principales deudores son Rusia, México y China, quienes absorben el $66 \%$ de la deuda total de estos países.

Con respecto al sector turístico, la selección de este grupo detenta tres de los grandes destinos turísticos del mundo: China, México y Rusia. Sin embargo, aunque los tres países figuran dentro de las quince naciones con mayor afluencia turística a nivel mundial, Rusia no forma parte de los quince países con mayores ingresos por turismo.

En cuanto a los indicadores del nivel de vida, debido a sus condiciones climáticas, $\mathrm{Ru}$ sia es el país con un mayor consumo per cápita de electricidad en el grupo (2 $309 \mathrm{kwh}$ ), pero si lo comparamos con Canadá, país con una relativa similitud climática, su consumo sólo alcanza el 26.5\% del consumo total de Canadá. Este hecho se debe en parte a las diferencias industriales, tecnológicas, de desarrollo y modernidad entre las dos economías. A Rusia, con su elevado consumo de fluidos eléctricos, le sigue Chile. En contraposición, los países que menos electricidad per cápita consumen son Filipinas (454 kwh, cifra que representa el 11\% del consumo de Rusia) y Perú (654 kwh), acumulado a sus bajos niveles de desarrollo.

Los habitantes de Perú y Filipinas son los que presentan los indicadores más bajos en el acceso a los medios modernos de comunicación. Por otro lado, las economías de este grupo que mayor número de usuarios de teléfono tienen son Chile, Malasia y México. Aunque en general la proporción de habitantes con posibilidades de adquirir una computadora personal y de ser usuario de Internet en este grupo es relativamente baja, es importante señalar que estos servicios se hallan en relativa expansión. A modo de ejemplo, podemos señalar a dos países que representan los polos de un continuum de mayor a menor en este conjunto: Chile y China. Para el primero en 1999 las personas que disponían de computadora eran 67 de cada mil habitantes, en 2000 este número creció a 82.3 personas, es decir, presentó un crecimiento de $23.5 \%$ con respecto al año anterior. En China, en 1999 sólo 12.2 de cada mil habitantes tenían una computadora personal; un año después el número creció $30.3 \%$ al alcanzar los 15.9 de cada mil personas.

\section{Economías con desarrollo bajo}

Dentro de la heterogeneidad de los países del APEC, Indonesia, Papúa Nueva Guinea y Vietnam son economías con desarrollo bajo. En general, se caracterizan por no contar con un sistema productivo complejo, sofisticado y moderno que les permita producir eficientemente grandes y variados volúmenes de bienes y servicios acordes con los tiempos y los requerimientos que la sociedad actual demanda. Lo anterior se refleja en la relativa baja producción interna del país, la cual permite que, en promedio, sus habitantes tengan un ingreso per cápita anual entre 2000 y 2830 dólares internacionales. Ello se refleja en la alta tasa de mortalidad infantil, en especial, en la de Papúa Nueva Guinea, con una tasa de 56 de cada mil niños nacidos. Más aún, la esperanza de vida en promedio de los ciudadanos de este grupo es de 65 años de edad, donde destaca una vez más Papúa Nueva Guinea con la tasa más baja: 59 años. A ello contribuye la baja proporción del gasto público que el gobierno destina a salud, el cual representa menos del 1\% del total del PIB. Llama la atención que Papúa Nueva Guinea, aunque destina mayores egresos públicos a salud, es la economía 
con los peores indicadores en el grupo. Probablemente influya el hecho de que la población no tiene a acceso a otro tipo de servicios, como son la alimentación y el agua potable, contribuyendo al detrimento de la salud. En Papúa Nueva Guinea el $26 \%$ de la población se encuentra desnutrido y sólo el $42 \%$ tiene agua entubada en sus casas, mientras que el $18 \%$ de la población carece de servicios de saneamiento adecuados (PNUD, 2002).

Con respecto a los años de escolaridad, ninguna de las economías de este grupo sobrepasa los 5 años de estudio. En Papúa Nueva Guinea el promedio de años de escolaridad es de sólo 2.9, presentando las mayores tasas de analfabetismo de los tres países a considerar (36.1\% de la población mayor de quince años). En Vietnam la tasa de analfabetismo es de $5.5 \%$, mientras que para Indonesia es de $13.1 \%$ (PNUD, 2002). Ello habla sobre la violación al derecho que tienen los niños a la educación y la falta de instituciones eficientes que promuevan e incentiven este derecho.

La distribución del ingreso es muy desigual en este grupo, aunque se acentúa más en Papúa Nueva Guinea, donde el $56.5 \%$ de la riqueza generada en el año 2000 pertenece al $20 \%$ más rico de la población, mientras que sólo el 4.5 del ingreso lo percibe el $20 \%$ más pobre de la ciudadanía. Este indicador concuerda con los otros parámetros del nivel de vida, como el consumo de electricidad, el acceso a líneas telefónicas o a teléfonos celulares, computadoras o Internet.

En cuanto a la estructura productiva de estas economías, la participación de la agricultura en el PIB es considerablemente alta, en 2000 representaba el $17 \%$ para Indonesia, el $26 \%$ para Papúa Nueva Guinea y el $24 \%$ para Vietnam. Sin embargo, con relación a 1990, ha disminuido para ensanchar la contribución del sector industrial y de servicios, indicando una gradual transformación productiva. Se distingue Vietnam, ya que en 1990 la agricultura era el segundo pilar más importante de la economía, pero en el transcurso de una década disminuyó 13 puntos porcentuales, aunque en
2000 este sector sigue empleando a la mayor parte de la población económicamente activa (67\%). Las principales industrias de Vietnam son las de alimentos procesados, prendas de vestir, calzado, construcción de maquinaria, minería, cemento, fertilizante, vidrios, frenos, petróleo, papel, carbón y acero. Los sectores industriales de Indonesia se centran en los mismos sectores que Vietnam, pero se agregan el petróleo, el gas natural, el textil y el turismo. Mientras que para Papúa Nueva Guinea sus industrias se basan en los recursos naturales, como almendra de coco, aceite de palma procesado, productos madereros, metales mineros (oro y plata), el petróleo crudo, la construcción y el turismo.

Durante la década de los noventa las tres economías presentaron altas tasas de inflación. Vietnam es el que tiene los mejores indicadores con respecto a la tasa de ahorro bruta como porcentaje del PIB (29.4\%), la de Indonesia es del 21.5 y la de Papúa Nueva Guinea del $17.7 \%$. Durante 2000, la inversión que impulsó la economía en Vietnam fue de 29.4 , mientras que para el resto fue de $18 \%$.

En el sector externo, el comercio total (exportaciones más importaciones) en 2000 fue de 126824 millones de dólares, lo que equivale al $2 \%$ del comercio total del resto de los miembros del APEC en ese mismo año. A excepción de Vietnam, los otros dos países presentan un superávit en la balanza comercial. Mientras el $57 \%$ de las exportaciones de Indonesia son productos manufacturados, en Papúa Nueva Guinea éstos representan el $2 \%$. Las exportaciones con relación al PIB son del 39 y $45 \%$, respectivamente. Por su parte, las importaciones equivalen al $31 \%$ para Indonesia y al $42 \%$ para Papúa Nueva Guinea, lo cual indica que al interior del país el comercio funge como una actividad de relativa importancia. En cuanto a la importación de alimentos, Papúa Nueva Guinea es la economía que más depende del exterior, al comprar el $18 \%$, en proporción al PIB.

Respecto al sector turístico, aunque en estos países se carezca de programas y planes específicos para atraer y promover el turismo, 
Cuadro 3

APEC: economías con desarrollo bajo, 2000

\begin{tabular}{|c|c|c|c|}
\hline & Indonesia & P. Nueva Guinea & Vietnam \\
\hline 1. Territorio, miles $\mathrm{km}^{2}$ & $1,811.6$ & 452.9 & 325.5 \\
\hline Forestal, porcentaje 2000 & 58 & 67.6 & 30.2 \\
\hline Promedio anual de deforestación 1990-2000 (\%) & 1.2 & 0.4 & -0.5 \\
\hline 2. Población, miles & $210,000.0$ & $5,000.0$ & $79,000.0$ \\
\hline Habitantes por $\mathrm{km}^{2}$, personas & 116 & 11 & 241 \\
\hline Esperanza de vida al nacer, años & 66 & 59 & 69 \\
\hline Tasa de mortalidad infantil, por 1000 nacidos vivos & 41 & 56 & 27 \\
\hline Mujer en la fuerza de trabajo (\%) del total & 40.8 & 42.2 & 48.9 \\
\hline Población mayor de 65 años, (\%) del total & 4.8 & 2.4 & 5.3 \\
\hline Promedio de años de escolaridad & 5 & 2.9 & N.D. \\
\hline \multicolumn{4}{|l|}{ 3. Economía } \\
\hline Ingreso Nacional Bruto (INB), mill. dls. & $119,900.0$ & $3,600.0$ & $30,400.0$ \\
\hline INB per cápita, dólares & 570.0 & 700.0 & 390.0 \\
\hline INB per cápita, dólares internacionales & $2,830.0$ & $2,180.0$ & $2,000.0$ \\
\hline Crecimiento promedio anual (1999-2000) (\%) & 4.8 & 0.3 & 5.5 \\
\hline \multicolumn{4}{|l|}{ Estructura del PIB (\%) (1990 y 2000) } \\
\hline Agricultura & 20 y 17 & 29 y 26 & 37 y 24 \\
\hline Industria & 38 y 47 & 30 y 44 & 23 y 37 \\
\hline Servicios & 42 y 36 & 41 y 30 & 40 y 39 \\
\hline Población activa, cifras en miles & $101,800.0$ & $2,500.0$ & $40,400.0$ \\
\hline Costo por trabajador en manufactura (dólares por año) (1995-1999) & $3,054.0$ & N.D. & 711.0 \\
\hline Inversión en la economía, porcentaje del PIB (\%) & 18 & 18 & 27 \\
\hline Tasa de ahorro bruta, porcentaje del PIB & 21.5 & 17.7 & 29.4 \\
\hline Inflación promedio anual, 1990-2000 (\%) & 15.5 & 7.9 & 15.4 \\
\hline \multicolumn{4}{|l|}{ 4. Sector externo } \\
\hline Exportaciones, millones de dólares & $62,124.0$ & $1,980.0$ & $14,450.0$ \\
\hline Exportaciones manufacturales como \% del total & 57 & 2 & N.D. \\
\hline Exportaciones en el PIB (\%) & 39 & 45 & N.D. \\
\hline Crecimiento promedio anual (\%) (1990-1999) & 8 & -7.4 & N.D. \\
\hline Importaciones, millones de dólares & $33,515.0$ & $1,120.0$ & $15,635.0$ \\
\hline Importación de alimentos, \% del total de importaciones & 10 & 18 & N.D. \\
\hline Importaciones en el PIB (\%) & 31 & 42 & N.D. \\
\hline Crecimiento promedio anual (\%) (1990-1999) & 4.2 & N.D. & N.D. \\
\hline Deuda externa (millones) & 141803 & 2604 & 12787 \\
\hline Turistas visitantes al año (miles de personas) & $5,064.0$ & 58.0 & $2,140.0$ \\
\hline Turistas al exterior (miles de personas) & N.D. & 106.0 & 168.0 \\
\hline Ingresos por turismo (millones de dólares) & $5,749.0$ & 76.0 & 86.0 \\
\hline \multicolumn{4}{|l|}{ 5. Otros indicadores del nivel de vida } \\
\hline Consumo de electricidad per cápita kwh (1999) & 345.0 & N.D. & 252.0 \\
\hline Líneas telefónicas y teléfonos móviles (2000), por 1000 habitantes & 69.0 & 15.0 & 54.0 \\
\hline Computadoras personales (2000), por 1000 habitantes & 9.9 & N.D. & 8.8 \\
\hline Usuarios de Internet (miles) & $2,000.0$ & 135.0 & 200.0 \\
\hline Periódicos por cada mil habitantes & 23 & 14 & 4 \\
\hline Gasto público en salud (\% del PIB) & 0.8 & 2.5 & 0.8 \\
\hline Coeficiente de Gini & 31.7 & 50.9 & 36.1 \\
\hline Ingreso o consumo del $20 \%$ más rico de la población (\%) & 41.1 & 56.5 & 44.5 \\
\hline Ingreso o consumo del $20 \%$ más pobre de la población (\%) & 9 & 4.5 & 8 \\
\hline
\end{tabular}

Fuente: Banco Mundial, World Bank Atlas 2002, World Development Indicators 2002, APEC Economic Outlook 2001 y 2002. www.brunei.gov.bn/about_brunei/economy.htm; www.stat.gov.tw; www.cia.gov/cia/publications/facbook/geos/bx.html.

Nota: N.D. = no disponible. 
los viajeros aprecian las bellezas naturales de esos lugares. Al interior, este sector es valorado por el ingreso de divisas y la creación de empleos en el país. Dentro de este grupo, las economías en las que el turismo proporciona mayores ingresos son Indonesia (5\%) y Papúa Nueva Guinea (2\%), mientras que para Vietnam esta actividad le genera un ingreso del $0.3 \%$ de su PIB.

\section{Conclusión}

Son dos las principales características de las economías del APEC:

1. Su heterogeneidad. Los factores que contribuyen a la formación de una sociedad, como los legales, culturales, los históricos, los religiosos y hasta los regímenes políticos no son compartidos en su mayoría. Los elementos, las circunstancias y las capacidades que permiten el desarrollo económico (con la transformación doméstica que ello implica) también difieren en cada una de las economías.

2. Su similitud al compartir intereses. El ampliar los beneficios económicos derivados del comercio y la inversión es la bandera en común que tienen estas economías.

\section{Notas}

1 Eurocentrista, confucionista, hinduista, budista, islamista, latinoamericanista, entre otros.

2 Clasificación basada en el ingreso per cápita, el cual se convierte a dólares internacionales en función de la paridad de poder adquisitivo (PPA). LaPPA se define como el número de unidades de la moneda de un país que se necesita para comprar en el mercado nacional la misma cantidad de bienes y servicios que se podrían obtener con un dólar en Estados Unidos. Ingreso menor a 2 995 dólares = países con nivel de desarrollo bajo; entre 2995 y 9265 = medio; mayor de 9266 = alto (World Bank Atlas, 2002: 48).
3 Esta postura se encuentra vinculada a la relación económica que existe entre la respuesta de la demanda y las variaciones de la renta, la cual explica que al aumentar el ingreso, la demanda de bienes de consumo primario (canasta básica) disminuye, y aumenta la proporción de su renta en el consumo de artículos de lujo (Fischer, Dornbursch y Schmalensee, 1990: 146-150).

4 Los parámetros van del cero al uno, conforme más cerca se encuentra del cero mayor igualdad existe, entre más cerca esté del uno mayor desigualdad económica.

5 Metas acordadas por los miembros delAPEC en 1994, en su encuentro en Indonesia.

6 http://www.apec.org.

7 http://www.world-tourism.org.

8 Aparte del problema del envejecimiento de la población, la sociedad japonesa se enfrenta al problema del bajo índice de fertilidad, estimado en 1.35 hijos por mujer (El Financiero, 22 de mayo de 2002).

\section{Bibliografía}

Cuevas Tello, Ana Bertha (2001) "Variables socioeconómicas de las economías delAPEC: 1999", en México y la Cuenca del Pacífico, vol 4, núm. 13, México: Departamento del Estudios del Pacífico, Universidad de Guadalajara.

Dornbusch, Rudiger y Stabley Fischer (1990) Macroeconomía. México: McGraw Hill, sexta edición.

Eisenstad, S. N. (1992) Estudios de modernización y teoría sociológica. Madrid: Alianza Editorial.

Huntington, Samuel (1985) Political modernization: America vs Europe. United State.

PNUD (2000) Human Development Report. Ediciones MundiPresa.

PNUD (2002) Informes sobre desarrollo humano: profundizar la democracia en un mundo fragmentado. Ediciones Mundi-Prensa.

Vera, Simona y Anne Thompson Feraru (1995) The Asia Pacific: political and economic development in a global context. Estados Unidos: Longman Publisher.

World Bank Atlas (2002) From the World Development Indicator. Washington. T: 